\title{
Design, manufacturing, alignment, and testing of the Back Telescope assembly of the MeteoSat Third Generation InfraRed Sounder (MTG- IRS)
}

Grégory Lousberg, Coralie De Clercq, Brunella Carlomagno, Jérémie Salazar, Virgile Monamy, et al.

Grégory P. Lousberg, Coralie De Clercq, Brunella Carlomagno, Jérémie Salazar, Virgile Monamy, Romain Vandoolaeghe, Jean-Marc Defise, Carlo Flebus, "Design, manufacturing, alignment, and testing of the Back Telescope assembly of the MeteoSat Third Generation InfraRed Sounder (MTG-IRS)," Proc. SPIE 11852, International Conference on Space Optics - ICSO 2020, 118521T (11 June 2021); doi: 10.1117/12.2599323

SPIE Event: International Conference on Space Optics - ICSO 2021, 2021, Online Only 


\section{International Conference on Space Optics-ICSO 2020}

Virtual Conference

30 March-2 April 2021

Edited by Bruno Cugny, Zoran Sodnik, and Nikos Karafolas
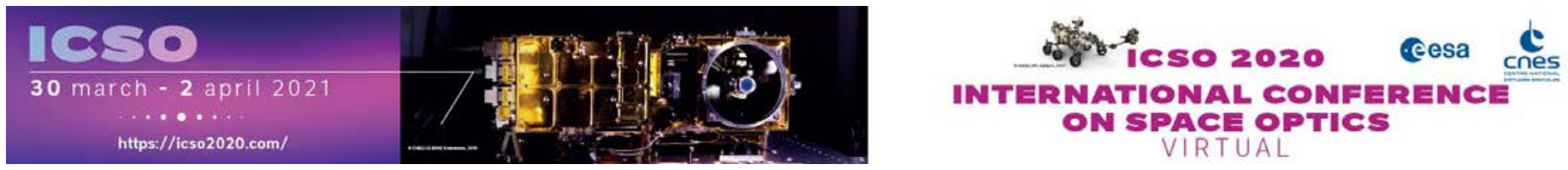

\section{Design, manufacturing, alignment, and testing of the Back Telescope assembly of the MeteoSat Third Generation InfraRed Sounder (MTG-IRS)}

\section{Cesa isopoceatings ecnes}




\title{
Design, manufacturing, alignment and testing of the Back Telescope Assembly of the MeteoSat Third Generation InfraRed Sounder (MTG-IRS)
}

\author{
Gregory P. Lousberg*, Coralie De Clercq, Brunella Carlomagno, Jérémie Salazar, Virgile Monamy, \\ Romain Vandoolaeghe, Jean-Marc Defise and Carlo Flebus \\ AMOS, Rue des Chasseurs Ardennais 2, 4031 Angleur, Belgium
}

\begin{abstract}
The Back Telescope Assembly (BTA) is one of the optical sub-systems composing the IRS instrument. It consists in an afocal off-axis four-mirror telescope whose primary function is to transfer the optical beam between the interferometer and cold detector units. The design, manufacturing and AIT activities are performed under the responsibility of AMOS (Liege, Belgium) with OHB System (Wessling, Germany) as the prime contractor of the IRS instrument.

In order to meet the challenging performance requirements, an innovative telescope design with four-mirror anastigmat optical configuration has been developed, using a full aluminum solution, i.e. mirrors and structure. It is manufactured and aligned under ambient conditions while it is operated close to $0^{\circ} \mathrm{C}$ in a non-uniform thermal environment. The aluminum structure of the BTA supports the 4 mirrors and is interfaced via 6 Titanium needles to the CFRP optical bench of the IRS instrument. The aluminum mirrors and the BTA structure are realized by the single-point diamond turning technique in AMOS manufacturing facilities.

In view of its qualification, the BTA models underwent an extensive testing campaign including: thermal-vacuum cycles, mechanical loads testing and model correlation, thermal balance and model correlation, measurement of the optical performances (wavefront error, magnification, optical axis orientation including its stability and pupil position) under ambient and operational conditions. The results of the tests allow to demonstrate the compliance of the BTA to the requirements.
\end{abstract}

Keywords: MTG, IRS, afocal telescope, aluminum optics, freeform mirror, thermal control, qualification test.

\section{INTRODUCTION}

The next generation of the European geostationary meteorological satellite constellation (Meteosat Third Generation MTG) is a joint program between ESA and EUMETSAT. The mission is based on a twin-configuration concept: an imaging (Flexible Combined Imager - FCI) and a sounder (InfraRed Sounder - IRS) satellites. The program will ensure continuity of the forecast services provided by the second generation of the weather satellites (MSG) and address future challenges in weather forecasting [1]. The first satellite (FCI) is currently planned to be launched by end 2022 while the IRS will be in-orbit one year later.

The instrument inside the IRS is a unique Fourier-transform based interferometer that will provide measurements of the time evolution of horizontal and vertical waper vapor structures in the atmosphere. The development of this state-of-theart instrument is led by OHB-System in Munich (Germany). One of the main IRS sub-systems is the Back Telescope Assembly (BTA). It consists in an optical relay system that transfers and scales the interferogram beam to the cold optics and focal planes. This sub-system is designed, manufactured and tested by AMOS in Liege (Belgium).

The BTA is an afocal telescope with a linear demagnification factor of $\sim 2.5$. The main requirements for the Back Telescope are listed in Table 1. In order to meet these challenging performance requirements, AMOS, in collaboration with OHB, developed an innovative aluminum telescope design. The full aluminum concept allows the optical design to scale with any temperature changes from the IRS environment without affecting the optical performances.

*gregory.lousberg@amos.be; www.amos.be 
Table 1. Main requirements and design drivers for the Back Telescope Assembly

\begin{tabular}{|c|c|}
\hline \multicolumn{2}{|c|}{ BTA REQUIREMENTS } \\
\hline \multicolumn{2}{|l|}{ Optical Requirements } \\
\hline \multirow[t]{2}{*}{ Wavelength range } & $4.44 \mu \mathrm{m}$ to $6.25 \mu \mathrm{m}$ (MWIR) \\
\hline & $8.26 \mu \mathrm{m}$ to $14.7 \mu \mathrm{m}(\mathrm{LWIR})$ \\
\hline Field of View (FoV) & $+/-2.2^{\circ}$ (square FoV) \\
\hline \multirow[t]{2}{*}{ Magnification } & $2.53-2.58$ along $\mathrm{Y}$ axis \\
\hline & $2.54-2.63$ along $Z$-axis \\
\hline Transmitted WaveFront Error & $<180 \mathrm{~nm}$ RMS \\
\hline In-orbit Line-of-Sight (LoS) stability & $<10 \mu \mathrm{rad}$ (1-sigma confidence level) \\
\hline \multicolumn{2}{|l|}{ Thermal Requirements } \\
\hline Thermal environment & $\begin{array}{l}\text { Non-uniform thermal environment with a } \\
\text { radiative coupling to a cold radiator }\left(T^{\circ} \text { close to - }\right. \\
\left.100^{\circ} \mathrm{C}\right)\end{array}$ \\
\hline Operational mirror temperature & $<0^{\circ} \mathrm{C}$ \\
\hline Mirror temperature stability & $<0.1 \mathrm{~K}$ over $15 \mathrm{~min}$ \\
\hline \multicolumn{2}{|l|}{ Mechanical Requirements } \\
\hline Mass & $<3.3 \mathrm{~kg}$ \\
\hline $1^{\text {st }}$ eigenfrequency & $>200 \mathrm{~Hz}$ \\
\hline Quasi-static load & $37 \mathrm{~g}$ \\
\hline
\end{tabular}

Several models of the Back Telescope have already been delivered to OHB for integration in the IRS instrument: the Mechanical and Thermal Dummy (MTD), the Qualification Model (QM) and the Proto-Flight Model (PFM). The second flight model (FM2) is currently starting its assembly and integration at AMOS premises. Several tests have been performed on these models and are summarized in Table 2.

This paper is organized as follows: the optical and mechanical design of the Back Telescope Assembly is described in Section 2, and the particular characteristics of the thermal control are discussed in Section 3. Section 4 is related to the manufacturing and alignment of the telescope, while Section 5 is dedicated to the qualification test campaign that has been performed on the Proto-Flight Model (PFM).

Table 2. BTA Test Matrix

\begin{tabular}{l|c|c|c|c}
\hline & Vibration Tests & Thermal cycles & Thermal Balance & Optical performances \\
\hline MTD & Shock + Random & & & \\
QM & Random + Sine & $\mathrm{X}$ & $\mathrm{X}$ & ambient conditions \\
PFM & Random + Sine & $\mathrm{X}$ & & operational conditions \\
FM2 & Random & & & ambient conditions \\
\hline
\end{tabular}




\section{OPTO-MECHANICAL DESIGN OF THE BACK TELESCOPE ASSEMBLY}

The optical design of the BTA is based on a Three-Mirror Anastigmat (TMA) configuration to which has been added a fourth folding mirror (see left panel of Figure 1). The telescope is afocal, i.e. a collimated beam at the entrance pupil is reimaged at the exit pupil as a collimated beam, and exhibits a linear de-magnification of 2.5 from entrance to exit pupil. Due to its stringent optical requirements, the first three mirrors are aspheric ones, while the folding mirror has a free-form shape that allows to control the magnification and the pupil position over the FoV. An unprotected gold coating (from Centre Spatial de Liege) is applied on the mirrors.

The BTA optical design is athermal, i.e. optical performances are maintained as the temperature varies uniformly in the structure. Both mirrors and structure are made of $6061 \mathrm{~T} 6$ aluminum. In particular, no additional Nickel-Phosphorus plating layer is added on the mirror surface (MWIR and LWIR applications). The BTA structure is realized from a single material block. It is extremely light-weighted and offers the required stiffness for the mirrors inside. Single-point diamond turning machine is used for mirrors and structure last manufacturing steps to provide the optical performance and the mechanical accuracy.

The aluminum structure of the BTA supports the 4 mirrors and is interfaced via 6 Titanium needles to the CFRP optical bench of the IRS instrument. These needles provide flexibility at the BTA interface in order to maintain the BTA alignment and hence, its optical performances, in case of interface deformations due to e.g. differential thermal expansion between aluminum and CFRP, distortion of the optical bench, interface flatness inaccuracies. On the other hand, the needles are also optimized to sustain the mechanical loads applied to the BTA and to be compatible with a first eigenfrequency requirement above a minimum of $200 \mathrm{~Hz}$. Details of the design of the 6-needles isostatic mount can be found in [2].

The CAD model of the BTA is shown on the right panel of Figure 1. Dimensions of the BTA are $400 \times 330 \times 200 \mathrm{~mm}$. Note that the thermal hardware is not represented on this view.
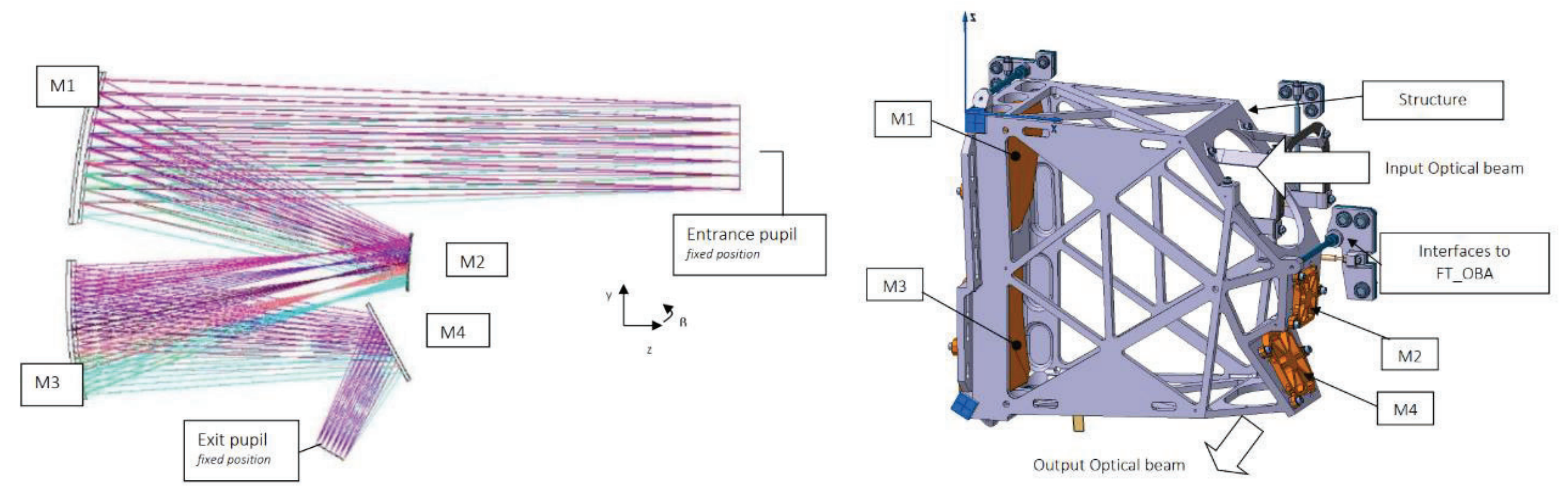

Figure 1. Left - BTA optical design. The entrance and exit pupils are not directly materialized in the BTA sub-system. Right - BTA mechanical design.

\section{THERMAL CONTROL STRATEGY}

The full aluminum concept allows the optical design to scale with any uniform temperature changes from IRS environment without affecting the optical performances. However, when submitted to non-uniform thermal environment, the aluminum structure is prone to develop large thermal gradients between the structure and the mirrors that can significantly impact the optical performances of the system due to the large coefficient of thermal expansion (CTE) of aluminum.

The thermal environment in which BTA is installed on the IRS instrument (see Figure 2) is highly non-uniform due to

1. Cold radiator on top of the BTA that is radiatively coupled to it through an MLI tunnel (radiator and MLI tunnel are not part of BTA)

2. Cold temperature from the cold optics and focal planes at BTA exit pupil,

3. Ambient temperatures from the interferometer assembly (at BTA entrance pupil) and optical bench on which is mounted the BTA. 


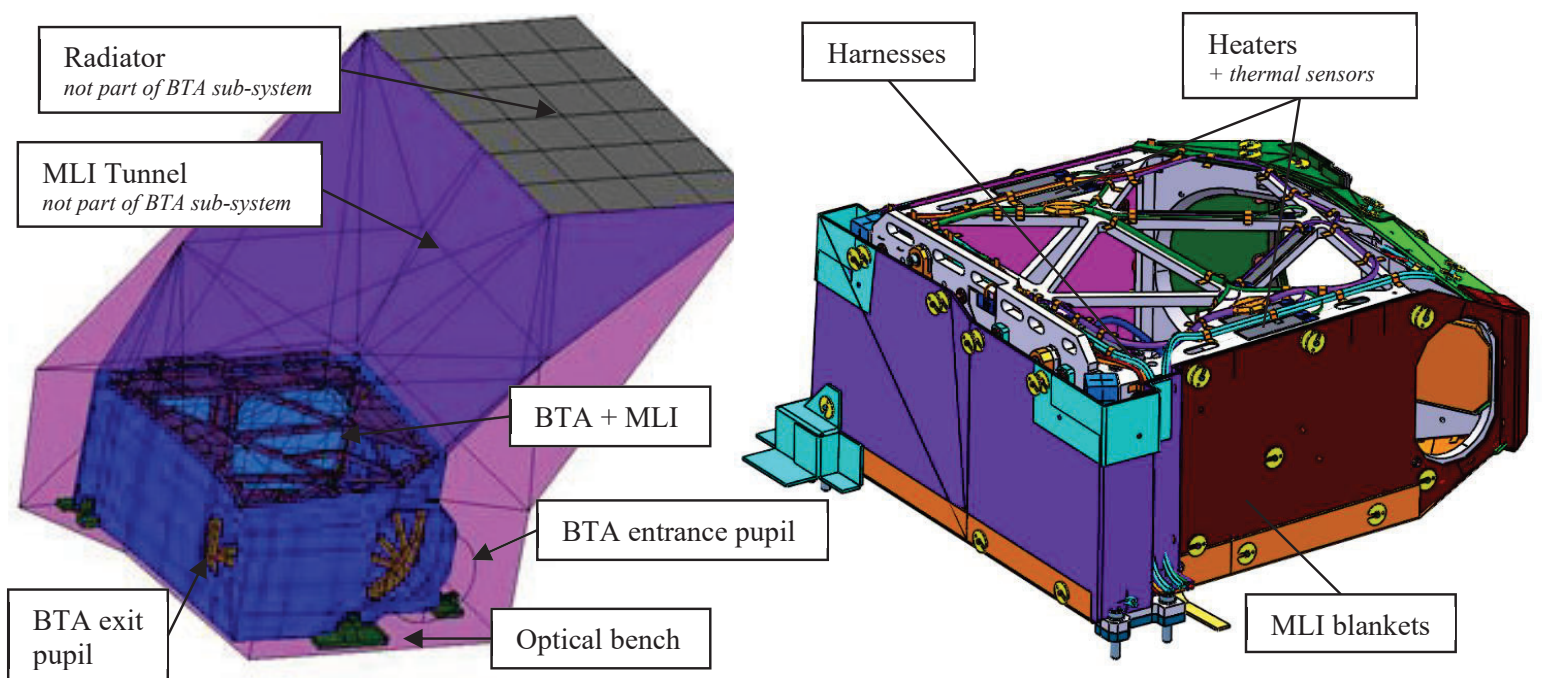

Figure 2. Left - Thermal environment in which BTA is located. Right - BTA with thermal hardware

In order to satisfy the optical performance requirements in the specified thermal environment, it has been necessary to implement thermal hardware on the BTA structure, i.e. heaters lines, thermal sensors and MLI insulation blankets (see Figure 2). The design of the BTA thermal control has been optimized to meet the optical performances requirements. To this aim, coupled structural, thermal, and optical performance (STOP) analyses have been performed. The coupling of the different models is performed with a tailored Matlab routine (EMIOS for End-to-end Modelling Interface for Optical Systems, AMOS internal software). Figure 3 shows an example of line-of-sight, pupil position and WFE computations during a $24 \mathrm{~h}$ orbit in hot conditions.
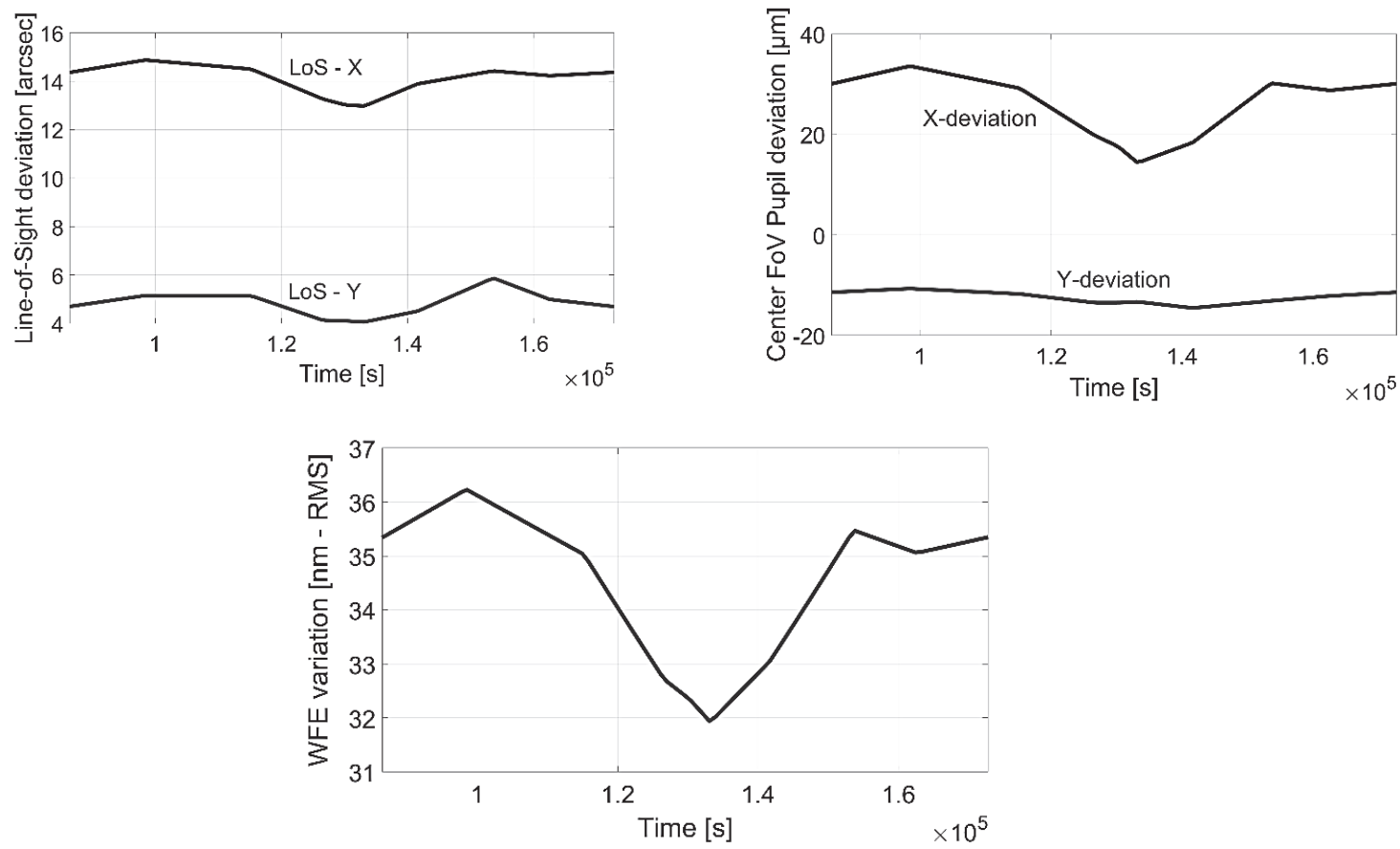

Figure 3. Optical performances of BTA under operational conditions (hot case). Line-of- sight / Entrance pupil position / Quadratic variation of the wavefront error 


\section{MANUFACTURING AND ALIGNMENT OF THE TELESCOPE}

The 4 BTA mirrors are made from Aluminum 6061T6. The required surface shape is obtained directly from diamond machining during the final step of the mirror manufacturing phase. The mirror surface qualities are verified by interferometric tests that required Computer-Generated Holograms (CGH). The deviations of the mirror surfaces from their nominal shapes are better than 50-70 nm RMS, and the mirror micro-roughnesses are of the order of $5 \mathrm{~nm}$ RMS. These performances are in line with both WFE and stray-light performance requirements. At the final step of mirror manufacturing, an unprotected gold coating layer is deposited on top of the mirror. A picture of a finished mirror is shown in Figure 4.

The BTA structure is manufactured from a Al $6061 \mathrm{~T} 6$ material block, using a conventional CNC machine. Because of the large amount of material that is removed, several heat treatments are applied to the structure during the manufacturing sequence. The interfaces for the mirrors and the alignment cubes are diamond-turned at the end of the machining process. The structure is treated with a black paint (MAP PU1) compliant with outgassing constraints and straylight requirements. The thermal hardware (composed of heaters, thermal sensors and cable harnesses) is mounted on dedicated area on the structure, as seen in Figure 4.

For the alignment of the telescope, the BTA is installed on a dedicated optical bench (see Figure 5) where the entrance and exit pupils are materialized. An interferometer sends a collimated beam toward the entrance pupil. The exit pupil features a mirror (mounted on a tip-tilt stage) that allows to measure the double-pass wavefront error and line-of-sight (LoS) of the telescope, and a camera equipped with a telecentric objective to characterize the position of the exit pupil of the telescope.

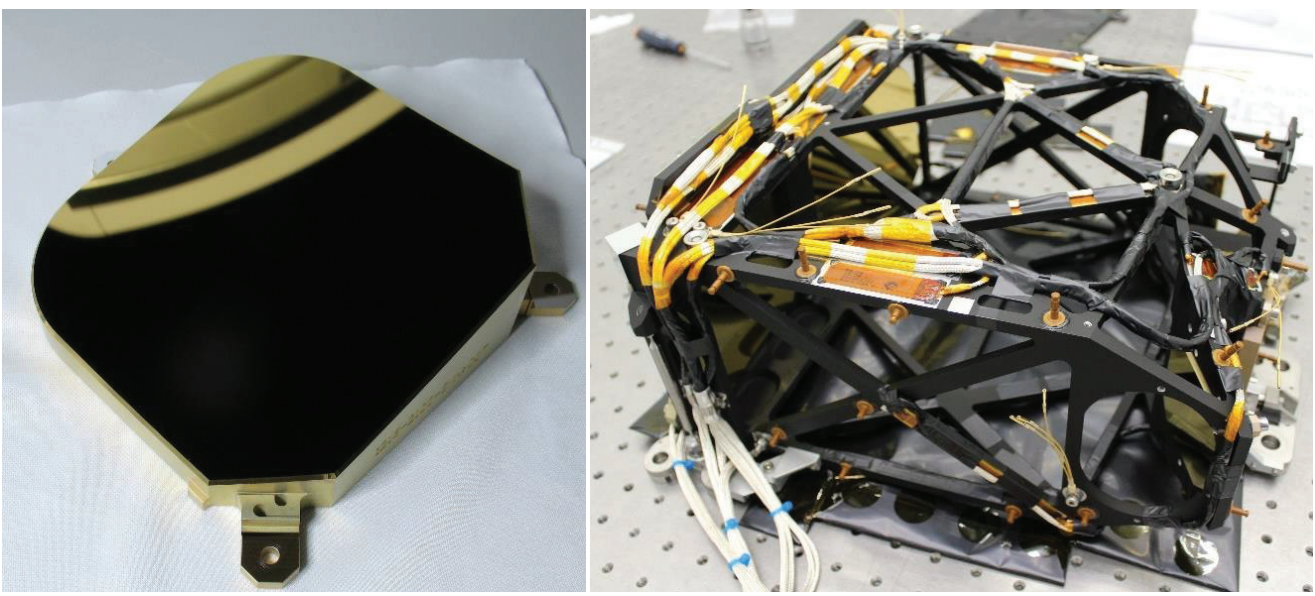

Figure 4. Left - M1 mirror with gold coating. Right - BTA structure with thermal hardware and mirrors

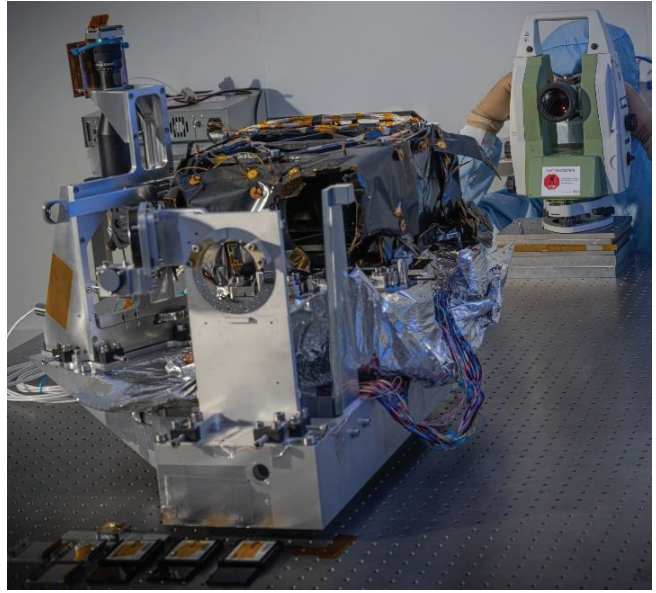

Figure 5. BTA on the optical bench during alignment and characterization under ambient conditions. 
The alignment of the telescope is straightforward thanks to the accuracy of the interfaces which are obtained by diamondturning. The telescope wavefront error is measured at the center of field-of-view (FoV) and is optimized by adapting the position of some mirrors. After few iterations, the WFE alignment is complete and the WFE is characterized in the complete FoV, as shown in Figure 6. Finally, the BTA position is adapted to meet the LoS and pupil positions requirements.

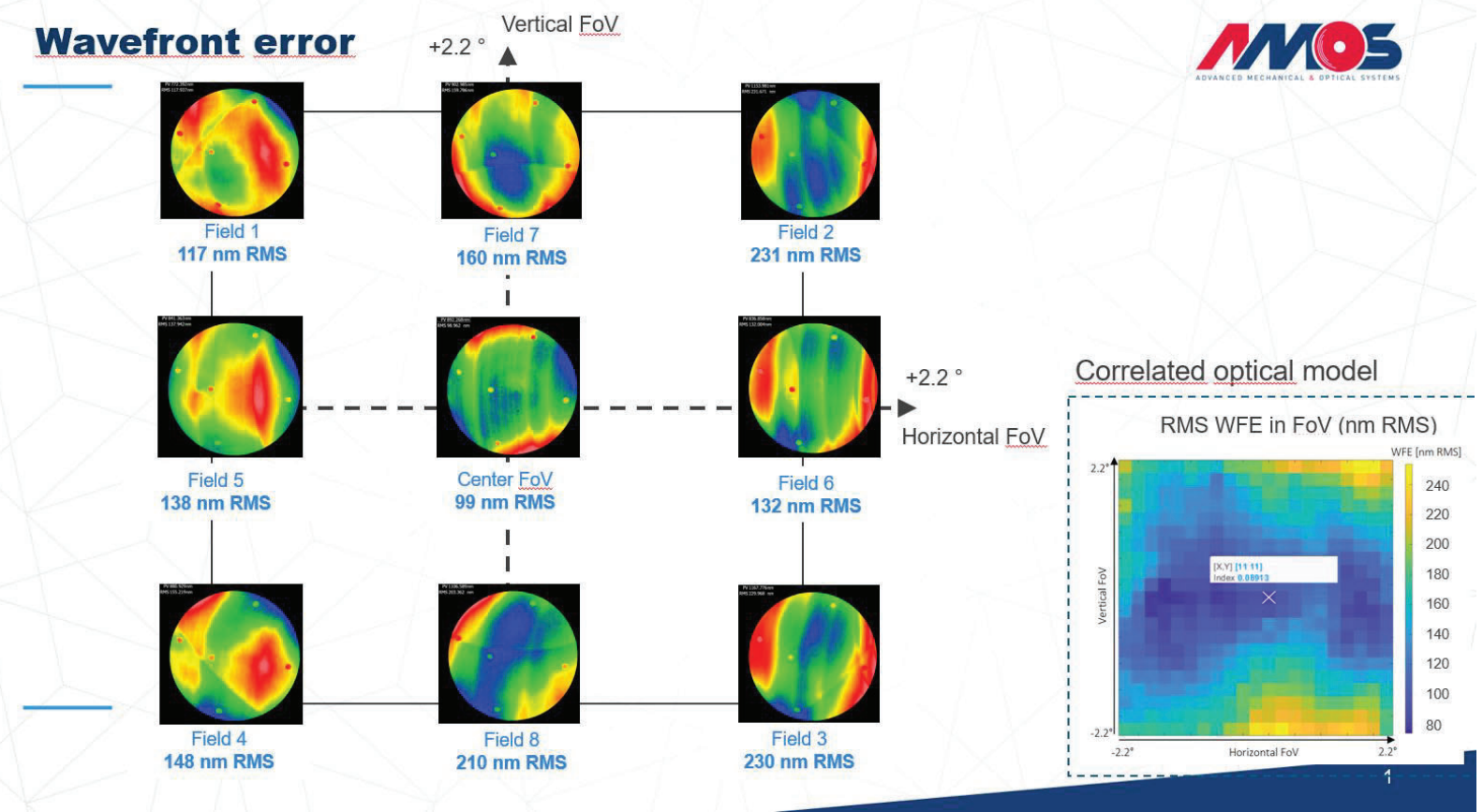

Figure 6. Transmitted single-pass wavefront error of the BTA after alignment under ambient conditions

\section{QUALIFICATION TEST CAMPAIGN}

The BTA model philosophy considers a proto-flight model approach, where the first flight model is fully tested and qualified according to the test flow that is sketched in Figure 7. The PFM test campaign took place from April to June 2020 in CSL facilities (Centre Spatial de Liège, Belgium).

Prior to PFM test campaign, two other BTA models were already tested and delivered to OHB. In particular, these models were used to correlate the mechanical and thermal models.

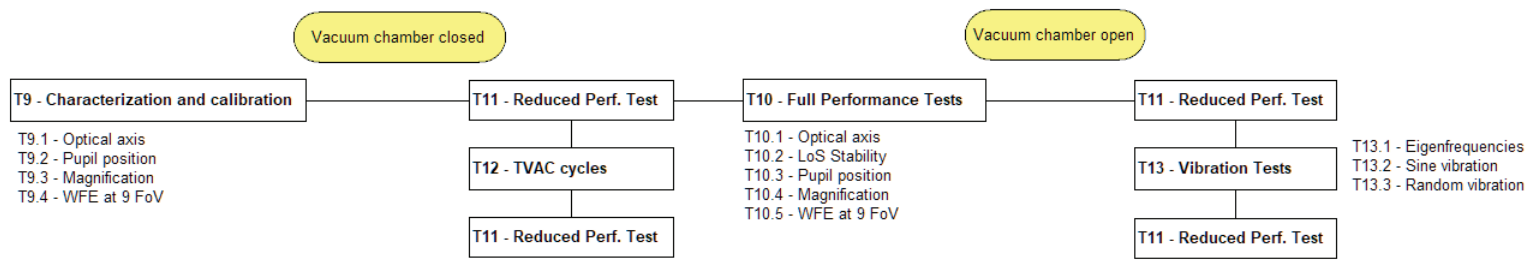

Figure 7. BTA PFM test flow 


\subsection{Vibration tests}

The vibrations tests were performed on a shaker installed in an ISO-5 cleanroom. For the vibration tests, the BTA is installed on a dedicated adapter plate. Several accelerometers are installed on the plate (for monitoring the excitation) and on the BTA structure (for measurements of the response of the BTA). The vibrations tests are performed with the MLI mounted on the BTA. A picture of the BTA installed on the CSL shaker is shown in Figure 8.

The following tests were performed (one test sequence per axis):

- Modal survey

- $\quad$ Sine vibrations (covering the quasi-static load of $37 \mathrm{~g}$ )

- $\quad$ Random vibrations (with notched inputs to not over-test the BTA)

Thanks to the structural model correlation already performed, the model predicts levels of accelerations seen by the accelerometers that are consistent with the measured accelerations on the PFM (see Figure 9).

A reduced set of optical measurements are also performed before and after the vibration test campaign. The BTA transmitted WFE and the optical axis orientation are stable (within the measurement accuracy limits) after exposure to the mechanical loads.

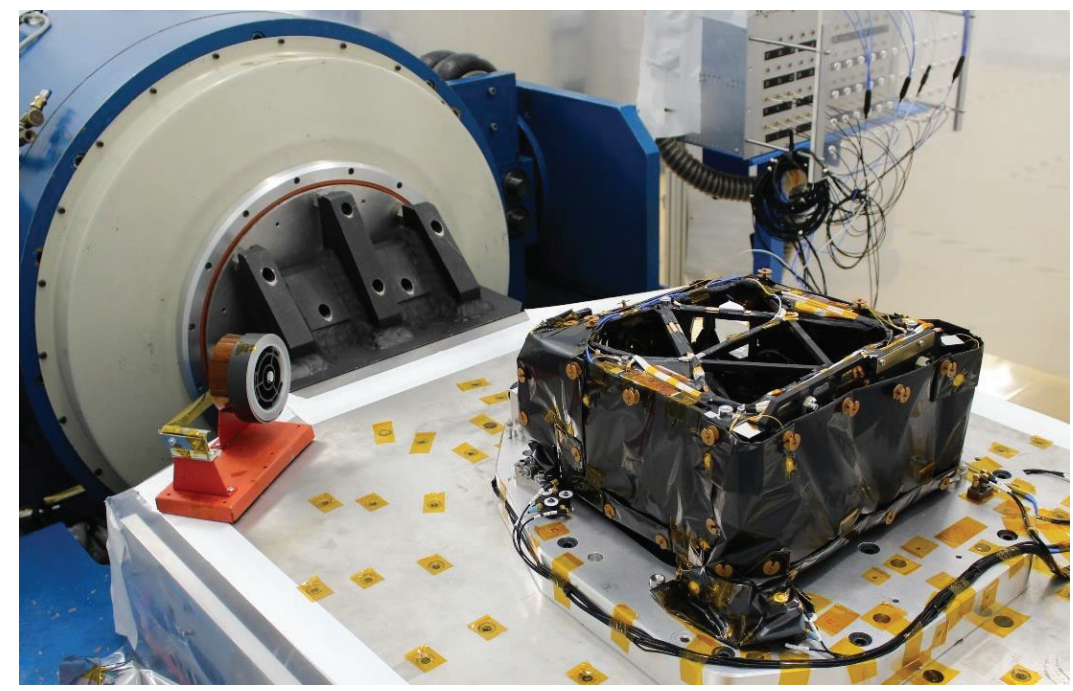

Figure 8. BTA installed on the shaker at Centre Spatial de Liege (CSL)
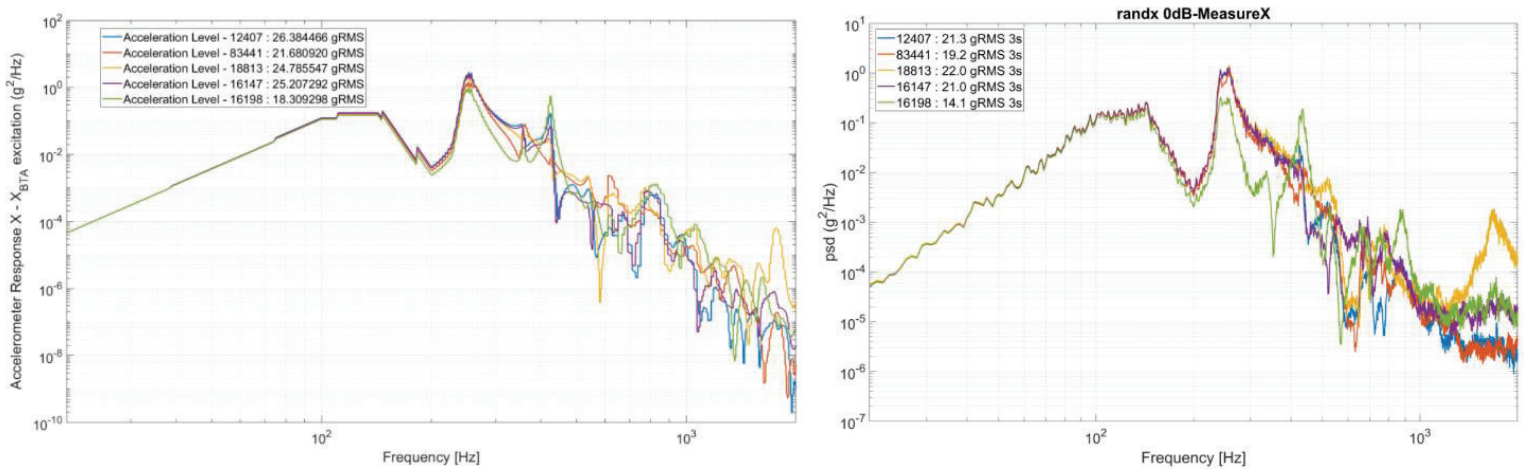

Figure 9. Example of random vibration measurements. Left - Predicted accelerations at the location of the accelerometers on BTA structure. Right - Measured acceleration levels. 


\subsection{Thermal cycles}

The BTA PFM was subjected to thermal cycles test under vacuum. Four cycles were applied (in line with a PFM approach), as depicted in Figure 10. The non-operational extreme temperatures are $-25^{\circ} \mathrm{C}$ and $+40^{\circ} \mathrm{C}$. The test was performed in the Focal-2 chamber at CSL with a dedicated thermal tent and a dedicated optical setup for optical performance measurement, so as to perform optical tests before and after the TVAC test, without opening the chamber. The WFE change (1 nm RMS) and LoS deviations ( 3 arcsec) after TVAC exposure are shown in in Figure 11 and are smaller than the test success criteria. The optical performances of the BTA are thus stable after exposure to thermal cycles.

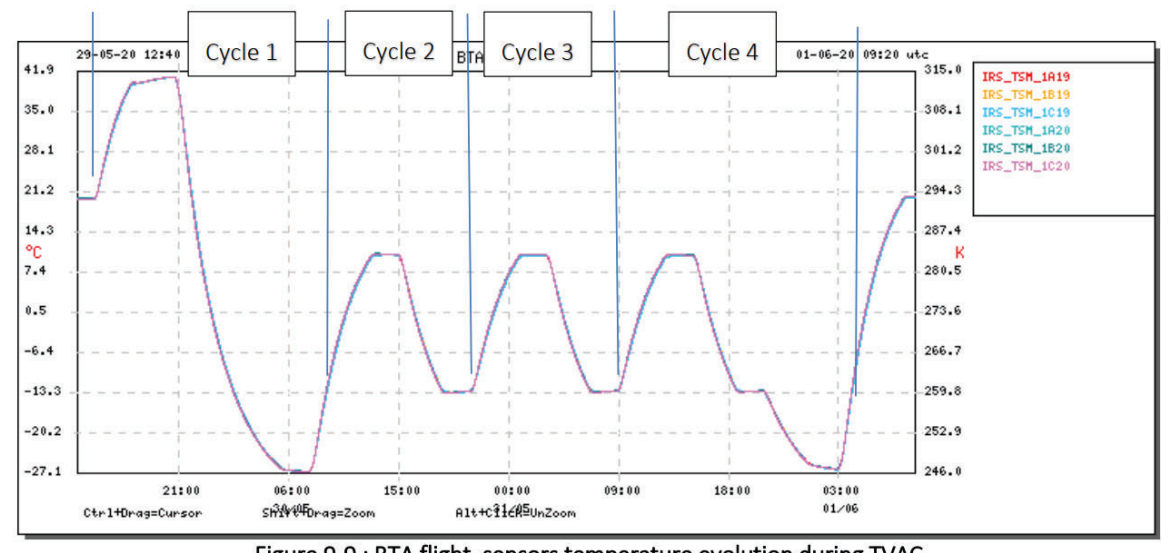

Figure 9-9 : BTA flight sensors temperature evolution during TVAC

Figure 10. BTA acceptance thermal cycles.

\section{Variation of line-of-sight before/after TVAC \\ Horizontal - 2 arcsec \\ Vertical -3 arcsec}

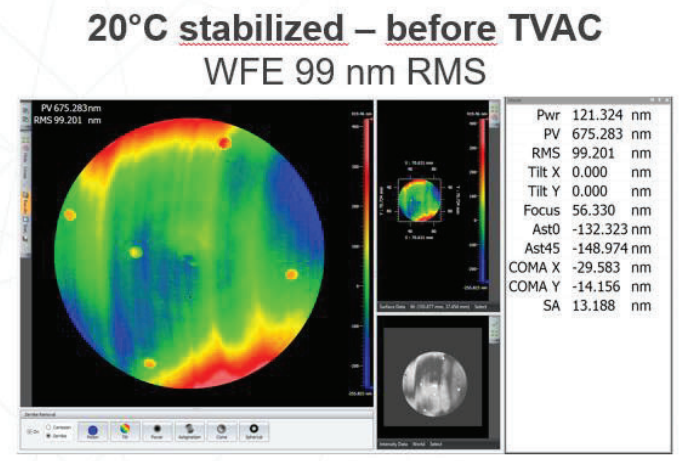

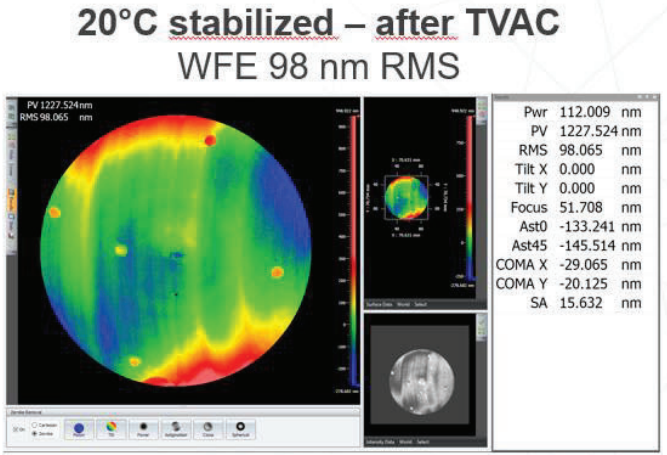

Figure 11. BTA optical performances variations after TVAC cycles 


\subsection{Thermal balance}

A thermal balance test was performed on the BTA qualification model (Figure 12). This test had the following purposes:

1- Provide key information for the correlation of the BTA thermal model, and hence, a better prediction of the BTA performances under operational conditions.

2- Verify the thermal performances of the BTA (stability of thermal control, power consumption, robustness of the controller)

Specific thermal configuration sequences were applied in the thermal tent so as to catch and highlight the different parameters of the thermal model (e.g. MLI efficiency, BTA thermal capacitance, parameters of the controller). The thermal panels were also set in representative operational conditions for the BTA in order to verify the thermal performances of the BTA.

The correlation of the thermal model has been performed after the tests. After correlation, the model is able to reproduce with a good accuracy the measured behavior of the BTA. As an example, Figure 13 shows the power consumption of both BTA heating lines in a specific situation in which the surrounding temperature had been raised wrt nominal conditions to entail heating lines cut-off. The model predictions (dashed lines) are consistent with the measured power consumptions (solid lines).
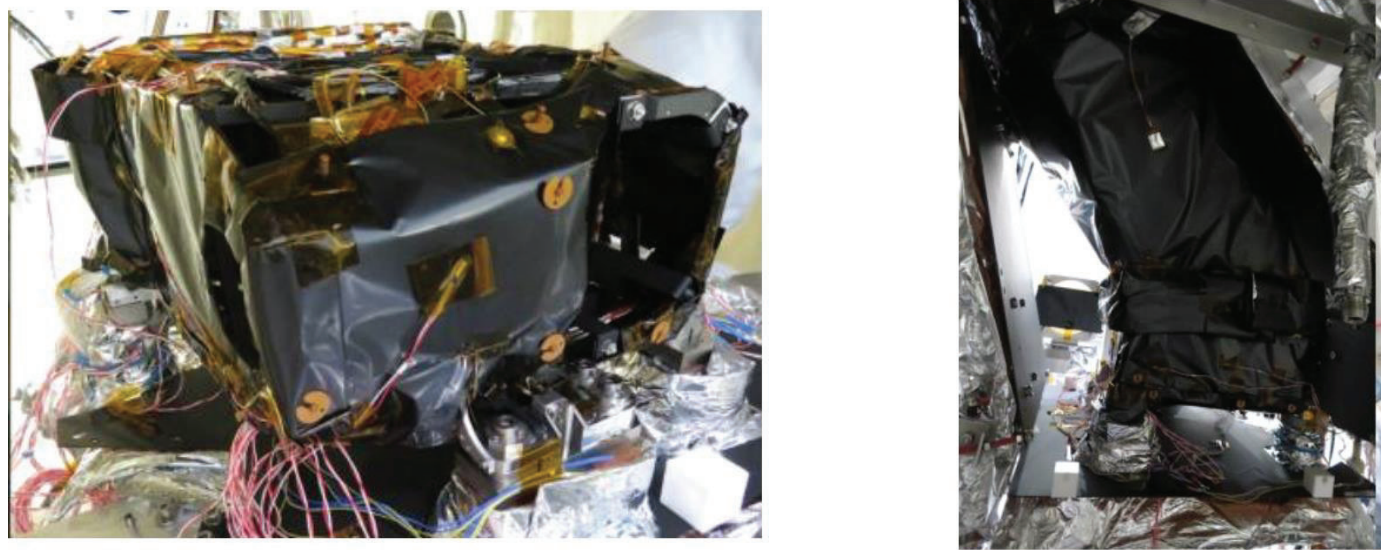

Figure 12. BTA in the thermal tent (only lower shroud is shown). Left - without MLI tunnel. Bottom - with MLI tunnel

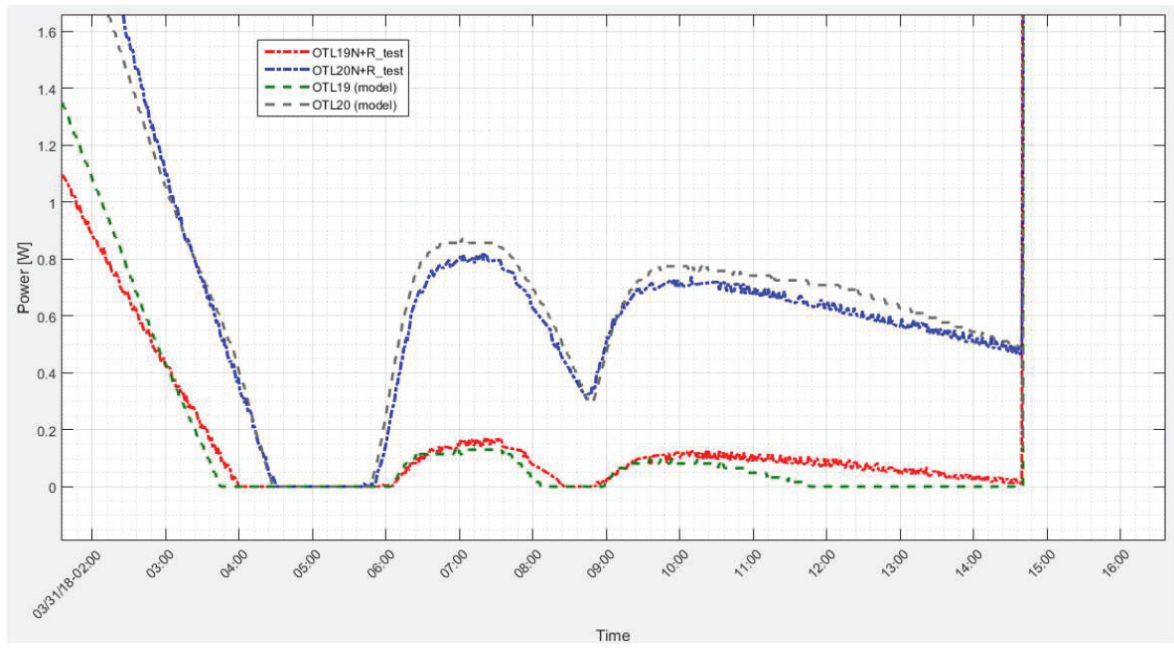

Figure 13. Example of the BTA thermal correlation based on the thermal balance test results. Power consumption of both operational thermal lines in case of line cut-off. 


\subsection{Optical performances under operational conditions}

Right after the TVAC test, the optical performances of the BTA were measured under operational conditions. The BTA was installed on its optical bench with the surrounding thermal panels (see Figure 14). The temperature on the thermal panels was set to be representative of the operational conditions of the BTA. Three cases were applied to verify the performance in steady (hot, cold) and transient conditions along a daily orbit.

For steady-state cases, the following optical performances were measured:

- $\quad$ Transmitted WFE in 9 positions in the FoV,

- Orientation of the optical axis at entrance and exit pupil planes (i.e. the Line-of-Sight of the BTA),

- Magnification, and

- $\quad$ Positions of the entrance and exit pupils.

The transient case was used to evaluate the stability of the line-of-sight during a 24h-orbit.

All optical performances are measured using a double-pass interferometric test setup with a collimated source provided by an interferometer located outside of the chamber and aligned to the entrance pupil.

The WFE performances under the COLD steady-state are reproduced in Figure 15 . The change of temperature from $20^{\circ} \mathrm{C}$ to $0^{\circ} \mathrm{C}$ caused a WFE difference of max $15 \mathrm{~nm}$ RMS, while the maximum WFE remains smaller than $250 \mathrm{~nm}$ RMS (approx. lambda/20). This demonstrates the very good athermal behavior of a full aluminum design. This is also observed with the magnification measurements that show a very good stability (at the level of 1\%) when going from ambient to operational conditions. The same conclusions were drawn from the measurements in HOT conditions.

As expected, the transition from ambient to operational conditions induces a shift of LoS orientation and pupil positions ( $\sim 25$ arcsec for the LoS shift and $\sim 100 \mu \mathrm{m}$ for the pupils). These shifts are compliant with the requirements and in line with the model predictions.

The results of the $24 \mathrm{~h} \mathrm{LoS} \mathrm{stability} \mathrm{test} \mathrm{are} \mathrm{shown} \mathrm{in} \mathrm{Figure} \mathrm{16.} \mathrm{The} \mathrm{time} \mathrm{signal} \mathrm{of} \mathrm{the} \mathrm{LoS} \mathrm{direction} \mathrm{is} \mathrm{reproduced} \mathrm{in} \mathrm{the}$ top part of Figure 16, while the cumulative statistics of the LoS deviation is shown in the bottom part. The stability of the LoS is better than $8 \mu \mathrm{rad}$ at 1 -sigma level, which meets the corresponding requirements.
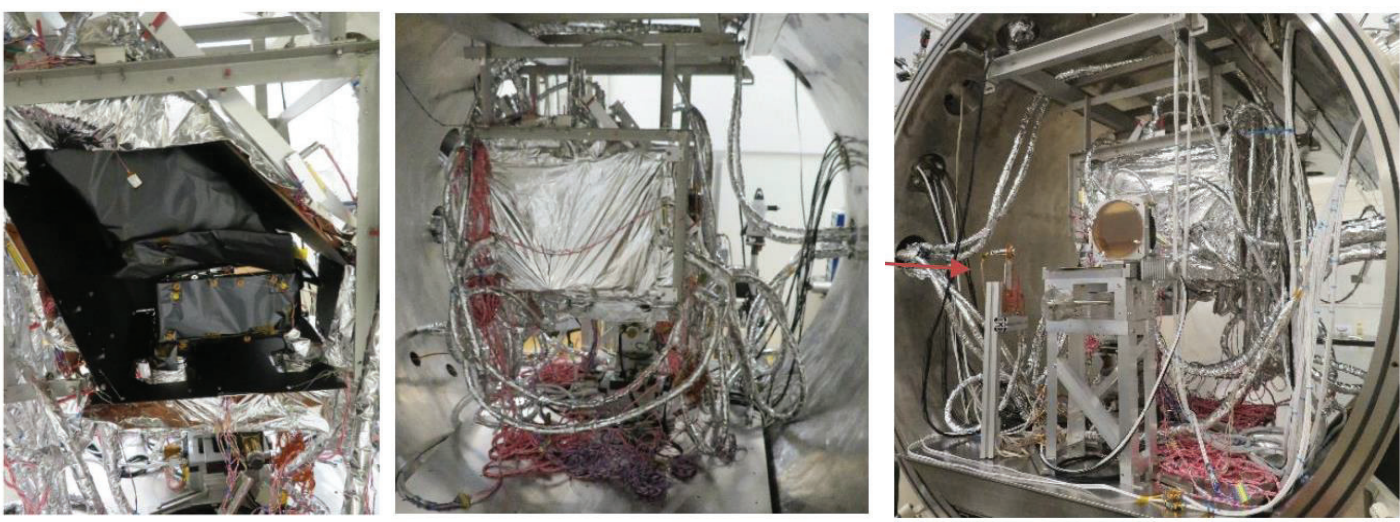

Figure 14. BTA installed in the thermal tent for tests under operational conditions at Centre Spatial de Liege (CSL). Left: BTA and the MLI tunnel on top of the BTA. Middle: thermal tent for the thermal operational condition simulation. Right: Thermal tent as seen from the entrance of the vacuum chamber with the opening in the thermal tent for the optical signal. 


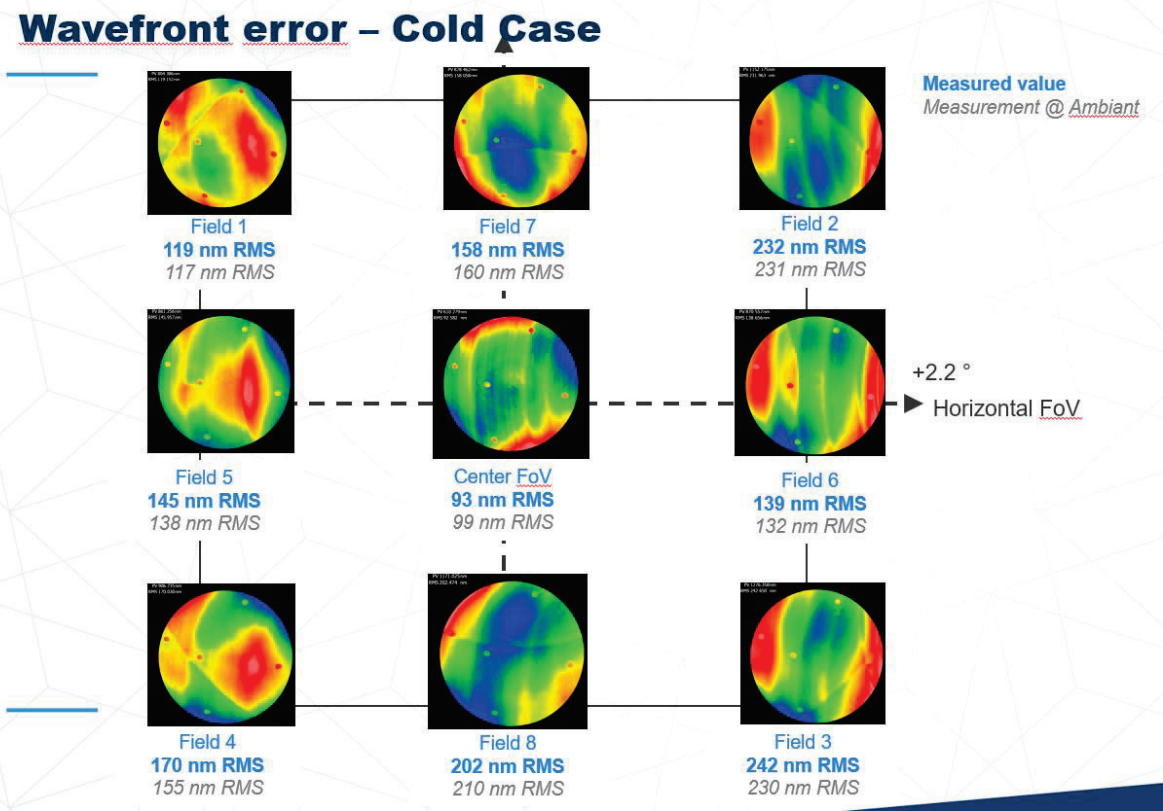

Figure 15. Transmitted single-pass wavefront error of the BTA in cold operational conditions
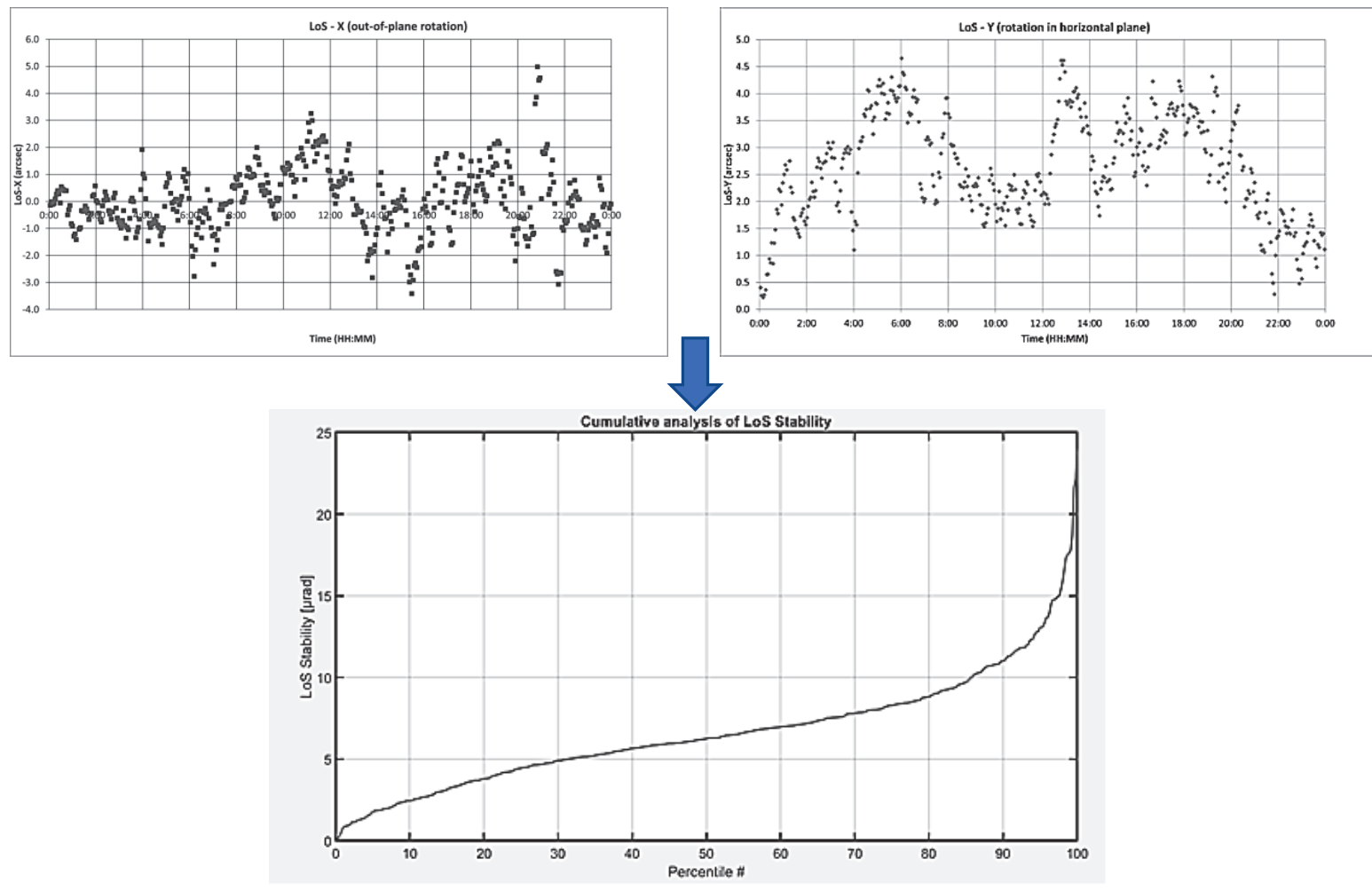

Figure 16 . LoS stability test during $24 \mathrm{~h}$ of operational conditions 


\section{CONCLUSIONS}

This paper presents the design, manufacturing and qualification testing of the Back Telescope Assembly (BTA) to be mounted inside the MTG-IRS instrument. The innovative full-aluminum telescope design that is interfaced via 6 Titanium needles to the optical bench has been successfully manufactured and integrated by AMOS.

In view of its qualification, the BTA models were subjected to an extensive testing campaign including: thermal-vacuum cycles, mechanical load testing and model correlation, thermal balance and model correlation, measurement of the optical performances (wavefront error, optical axis orientation including its stability and pupil position) under ambient and operational conditions. The results of the tests have demonstrated the very good behavior of the system under operational conditions and its compliance to the demanding requirements.

The following key performances are highlighted:

1. The athermal design with aluminum mirrors and structure allows to almost maintain the transmitted WFE and magnification under all operational conditions, while there is a temperature difference of $20^{\circ} \mathrm{C}$ wrt the ambient conditions under which the BTA is aligned, and

2. The very good stability of the LoS under operational conditions which benefits from an adequate mechanical and thermal design.

\section{ACKNOWLEDGEMENT}

The authors would like to acknowledge OHB System (Wessling, Germany), Thales Alenia Space (Cannes, France), ESA and the Belgian Science Policy Office (BELSPO) for their support during the project.

\section{REFERENCES}

[1] Bézy, J. L., Aminou, D., Bensi, P., Stuhlman, R., Tjemkes, S. and Rodriguez, A., "Meteosat Third Generation The Future European Geostationary Meteorological Satellite," ESA Bulletin, No 123, August 2005, pp. 28-32

[2] Freudling, M., Klammer, J., Lousberg, G., Schumacher, J. M. and Körner, C, " New isostatic mounting concept for a space born Three Mirror Anastigmat (TMA) on the Meteosat Third Generation Infrared Sounder Instrument (MTG-IRS)," Proc. SPIE Vol. 9912, 99121F, (2016). 